\title{
Coefficient of thermal expansion of carbon nanotubes measured by Raman spectroscopy
}

Libo Deng, Robert J. Young, lan A. Kinloch, Rong Sun, Guoping Zhang, Laure Noé, and Marc Monthioux

Citation: Appl. Phys. Lett. 104, 051907 (2014); doi: 10.1063/1.4864056

View online: https://doi.org/10.1063/1.4864056

View Table of Contents: http://aip.scitation.org/toc/apl/104/5

Published by the American Institute of Physics

\section{Articles you may be interested in}

Carbon nanotube composites for thermal management

Applied Physics Letters 80, 2767 (2002); 10.1063/1.1469696

Dominant role of tunneling resistance in the electrical conductivity of carbon nanotube-based composites

Applied Physics Letters 91, 223114 (2007); 10.1063/1.2819690

Interface effect on thermal conductivity of carbon nanotube composites

Applied Physics Letters 85, 3549 (2004); 10.1063/1.1808874

Raman Spectrum of Graphite

The Journal of Chemical Physics 53, 1126 (1970); 10.1063/1.1674108

Thermal and Electrical Conductivity of Graphite and Carbon at Low Temperatures

Journal of Applied Physics 15, 452 (1944); 10.1063/1.1707454

Temperature dependence of the Raman spectra of carbon nanotubes

Journal of Applied Physics 84, 4022 (1998); 10.1063/1.368585

\section{Scilight}

Sharp, quick summaries illuminating the latest physics research

\section{Sign up for FREE!}

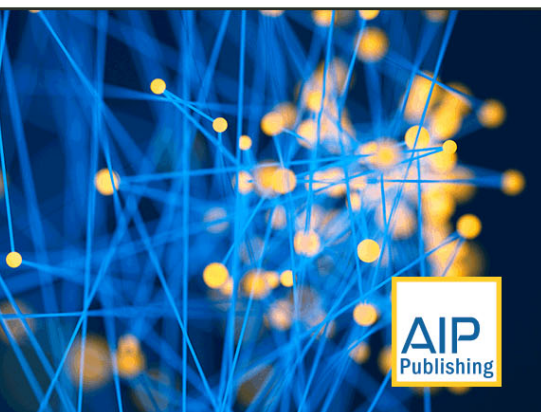




\title{
Coefficient of thermal expansion of carbon nanotubes measured by Raman spectroscopy
}

\author{
Libo Deng, ${ }^{1,2}$ Robert J. Young, ${ }^{2, a)}$ lan A. Kinloch, ${ }^{2}$ Rong Sun, ${ }^{1}$ Guoping Zhang, ${ }^{1}$ \\ Laure Noé, ${ }^{3}$ and Marc Monthioux ${ }^{3}$ \\ ${ }^{1}$ Shenzhen Institute of Advanced Technology, Chinese Academy of Sciences, Shenzhen 518055, China \\ ${ }^{2}$ Materials Science Centre, School of Materials, University of Manchester, Oxford Road, \\ Manchester M13 9PL, United Kingdom \\ ${ }^{3}$ CEMES, UPR 8011 CNRS, Toulouse, France
}

(Received 15 November 2013; accepted 27 December 2013; published online 4 February 2014)

\begin{abstract}
The coefficient of thermal expansion (CTE) of peapod-derived double-walled carbon nanotubes and their host empty single-walled carbon nanotubes (SWCNTs) was determined using Raman spectroscopy. This was performed by measuring the dependence of Raman band frequency of the nanotubes in epoxy resin matrix composites and considering the effects of both the strain and temperature on the Raman bands. Both types of nanotubes show positive thermal expansion at room temperature of around $+2 \times 10^{-5} \mathrm{~K}^{-1}$, and the CTE of the SWCNTs was unaffected by the introduction of the inner wall nanotubes. It was also demonstrated that the temperature-induced Raman band shifts can be used to determine both the CTE and glass transition temperature of the matrix polymers. ㅇ 2014 AIP Publishing LLC. [http://dx.doi.org/10.1063/1.4864056]
\end{abstract}

Understanding the thermal properties of carbon nanotubes (CNTs), particularly the coefficient of thermal expansion (CTE), is crucial for a range of CNT-based applications. ${ }^{1}$ Several theoretical approaches such as molecular dynamics and density-functional theory have been proposed to predict the CTE of nanotubes. There are, however, large variations in thermal behaviour of nanotubes derived using the different theories. Jiang et al. estimated the CTE of single-walled carbon nanotubes (SWCNTs) to be positive and $\sim+2 \times 10^{-6} \mathrm{~K}^{-1}$ at $300 \mathrm{~K}$ (all CTEs in this study are axial unless otherwise stated), ${ }^{2} \mathrm{Kwon}$ et al. determined a value of the CTE for SWCNTs to be negative and $\sim-1.1 \times 10^{-5} \mathrm{~K}^{-1}$ at the same temperature, ${ }^{3}$ and Schelling et al. showed that the CTE of nanotubes is dependent on their chirality. ${ }^{4}$ On the other hand, very limited experimental studies have been carried out so far due to the challenge in nanoscale experiments. ${ }^{1}$ Maniwa et al. determined the CTE of a SWCNT bundle to be $(+7.5 \pm 2.5) \times 10^{-6} \mathrm{~K}^{-1}$ between 300 and $900 \mathrm{~K}$ using an X-ray diffraction (XRD) method. ${ }^{5,6}$

Raman spectroscopy is a powerful technique to investigate a range of carbon nanomaterials. ${ }^{7}$ It has been used to determine the Young's modulus and thermal conductivity of CNTs and graphenes, ${ }^{8-10}$ based on the fact that Raman bands are sensitive to the stress (and strain) and temperature. ${ }^{11}$ It is proposed the CTE of CNTs can also be measured by this technique. When the temperature of a polymer nanocomposite containing CNTs is changed, the mismatch of CTE between the matrix and CNTs induces strain on the latter. By following the Raman band position, the thermal-induced strain can be evaluated and thus the CTE can be determined.

In this present study, double-walled carbon nanotubes (DWCNTs) and their host SWCNTs were incorporated into epoxy resins. We first measured the temperature dependence of the Raman $\mathrm{G}^{\prime}$-band positions for the nanotube powders.

\footnotetext{
${ }^{\text {a) }}$ Author to whom correspondence should be addressed. Electronic mail: Robert.Young@manchester.ac.uk
}

The dependences of $\mathrm{G}^{\prime}$-band positions upon the strain and temperature for the nanotubes in composites were then investigated with composites prepared under identical conditions. The CTEs were determined taking into account of the effects of strain and temperature on the Raman band. Using the same composite matrix eliminates the effects of orientation, dispersion and interfacial adhesion (which are known to have significant effects ${ }^{12}$ ) upon the Raman response, and hence allows an accurate determination of the CTE of the nanotubes.

The SWCNTs were prepared via the electric-arc method (Nanocarblab); the DWCNTs were prepared by coalescence of $\mathrm{C}_{60}$ that encapsulated in the SWCNTs, and the preparation procedures were described in our previous work. ${ }^{13}$ PRD-166 alumina/zirconia fibers with a CTE of $+9 \times 10^{-6} \mathrm{~K}^{-1}$ at $300 \mathrm{~K}$ (Ref. 14) were supplied by DuPont and were used to determine the coefficient of thermal expansion of the epoxy resin matrix. The epoxy resin/CNT composites were prepared using a two-part epoxy system consisting of a hardener (Araldite HY 5052) and a resin (Araldite LY5052) following the method described in our previous work. ${ }^{15}$ The weight fraction of the nanotubes in the composites was approximately $0.1 \%$. To prepare epoxy resin/PRD-166 composites, a single PRD-166 fiber was placed on an epoxy beam and coated with a thin layer of epoxy resin and the curing conditions were the same as those for the epoxy/CNT composites.

Raman spectra were obtained using a Renishaw 2000 system with a $633 \mathrm{~nm}$ laser. For deformation testing, the epoxy resin beam on which the epoxy resin/filler composites were deposited was inserted into a four-point bending rig and placed on the Raman microscope stage. ${ }^{9}$ The surface strain was measured using a resistance strain gauge. It was assumed that the strain in the nanotubes was the same as in the epoxy resin, and also the epoxy beam, as given by the strain gauge. For in-situ Raman measurements during heating, samples were placed in a chamber equipped with an optical window and a heating stage (programmable hot stage TS 1500). The temperature was increased in steps of 


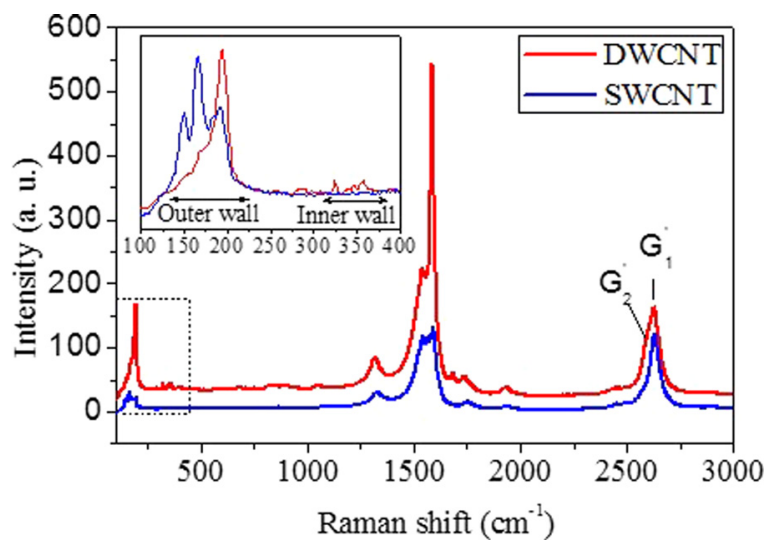

FIG. 1. Raman spectra of DWCNTs and the host SWCNTs.

$10 \pm 1 \mathrm{~K}$. Spectra were collected $2 \mathrm{~min}$ after each temperature of interest was reached.

Fig. 1 shows typical Raman spectra of the DWCNTs and SWCNTs powders. In the low-frequency Raman spectrum of DWCNTs, multiple radial breathing mode (RBM) peaks can be seen. The peaks below $200 \mathrm{~cm}^{-1}$ correspond to nanotubes with diameters greater than $1.24 \mathrm{~nm}$ according to the $\omega_{\mathrm{RBM}}=248 / d_{\mathrm{t}}$ (where $\omega_{\mathrm{RBM}}$ is the frequency of RBM peak and $d_{\mathrm{t}}$ is the nanotube diameter) relation ${ }^{16}$ and are attributed to the outer wall nanotubes, while the RBM peaks beyond $260 \mathrm{~cm}^{-1}$ originate from the inner wall nanotubes, with diameters ranging from $0.65 \mathrm{~nm}$ to $0.95 \mathrm{~nm}$. The $\mathrm{G}^{\prime}$-band of DWCNTs clearly splits into two peaks, as can be seen from Fig. 1. It is straightforward to assign the higher-frequency peak (denoted as $\mathrm{G}_{1}^{\prime}$ herein) to the outer tubes and the lower-frequency peak $\left(G_{2}^{\prime}\right)$ to the inner tubes, due to the strong diameter dependence of $\omega_{\mathrm{G}^{\prime}}{ }^{13}$

The nanotubes were dispersed in an epoxy resin matrix and the epoxy/CNT composites were deformed with a fourpoint bending rig to investigate both the interfacial adhesion between the nanotubes and the polymer matrix, and the interaction between the concentric graphene layers in the DWCNTs. Figs. 2(a) and 2(b) are schematic diagrams showing the distribution of nanotubes in the matrix, and Fig. 2(c) illustrates the measured strain-induced shifts of the $\mathrm{G}^{\prime}$-band during deformation of the composites. The linear fit in the $0 \%-0.4 \%$ range gives the Raman band shift rate (also denoted as the strain coefficient herein). In Fig. 2(c), the most striking feature is that the $G^{\prime}$ shift rate of the inner wall $\left(G_{2}^{\prime}\right)$ is significantly lower than that of the outer wall $\left(\mathrm{G}_{1}^{\prime}\right)$, which has been explained as a consequence of the low efficiency of stress transfer from the outer wall to the inner wall. ${ }^{13}$ Theoretical work showed that during the preparation of DWCNTs through a pea-pod route, the encapsulated $\mathrm{C}_{60}$ fullerenes in the SWCNTs coalescence into corrugated inner tubes with multiple intra-tube junctions ${ }^{17,18}$ Such loose fitting and short inner tubes might not allow a full development of stress in the inner tubes.

The $\mathrm{G}^{\prime}$-band shift rates shown in Fig. 2(c) were obtained for composites containing randomly distributed nanotubes with no preferred orientation (see Fig. S1 in the supplemental material ${ }^{19}$ ). The Raman signals were the results of contributions from nanotubes lying in all directions with respect to the laser polarization and the strain, and the nanotubes at different angles respond differently to the external strain. The overall band shift can be considered as an intensity-weighted average of band shift for nanotubes lying at all angles. Therefore, the shift rate for nanotubes parallel to the strain, $S_{0}$, can be derived considering the effect of the polarization-dependence of Raman spectrum and the Poisson's contraction effect such that ${ }^{20}$

$$
S_{0}=\frac{\int_{0}^{\pi} \cos ^{4} \theta \times\left(\cos ^{2} \theta-\nu \sin ^{2} \theta\right) \mathrm{d} \theta}{\int_{0}^{\pi} \cos ^{4} \theta \mathrm{d} \theta} \times S(0)
$$

where $v$ is the Poisson's ratio of the matrix, $\theta$ is the angle between the nanotube and the strain direction, and $S(0)$ is the shift rate measured for a randomly distributed composite when the strain axis is parallel to the laser polarization. Substituting $v=0.35$ for the epoxy resin into Eq. (1) gives $S_{0}=1.3 \times S(0)$. This suggests that if all nanotubes were aligned perfectly along the strain direction (Fig. 2(b)), the shift rates would be about $30 \%$ higher than those shown in Fig. 2(c). The dependences of Raman band upon the strain for perfectly aligned nanotubes derived using Eq. (1) are shown in Fig. 2(d), and the values for $S_{0}$ estimated using Eq. (1) are listed in Table I.

The nanotube powders were heated in an air atmosphere using the hot stage. Fig. 3(a) shows the dependence of
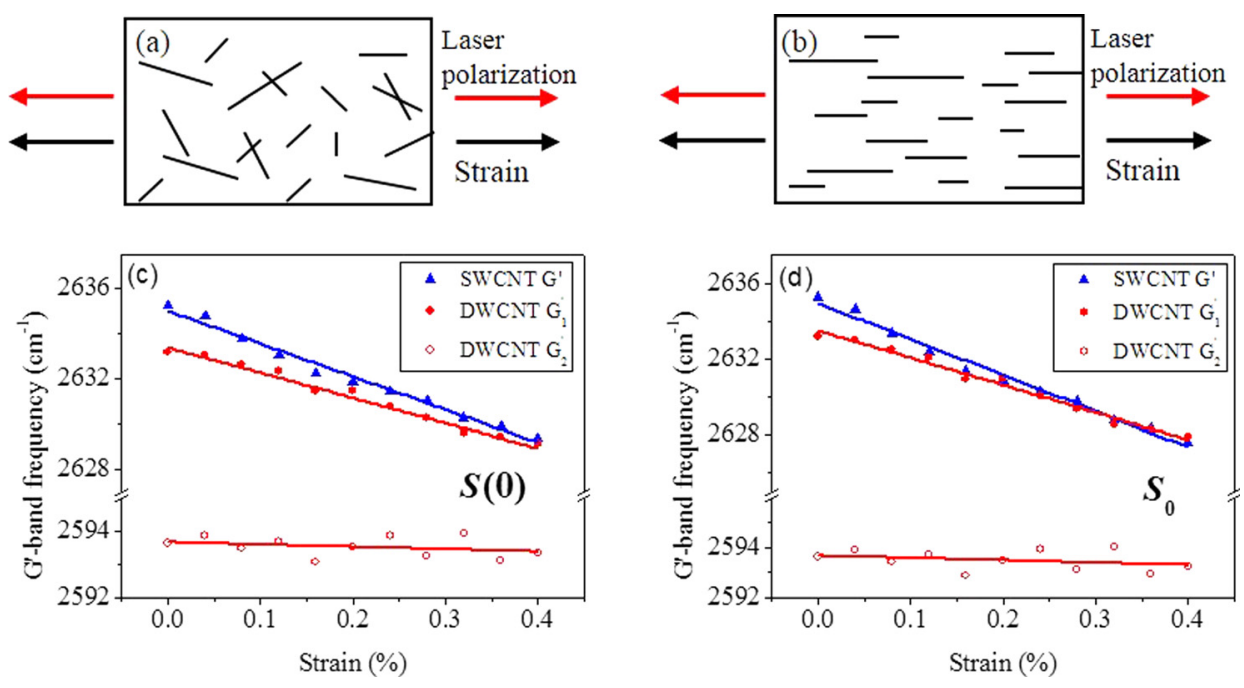

FIG. 2. Schematic diagrams of nanocomposites containing (a) randomly distributed and (b) perfectly aligned nanotubes; (c) Measured $\mathrm{G}^{\prime}$-band frequencies as a function of strain for the composites in which a random distribution of the nanotubes was found and (d) derived response of the $\mathrm{G}^{\prime}$-band to the strain for perfectly aligned nanotubes. 
TABLE I. The strain and temperature coefficients of the Raman bands of the fillers and the CTEs calculated for the CNTs.

\begin{tabular}{lrrrr}
\hline \hline Material & $S_{0}\left(\mathrm{~cm}^{-1} / \%\right)$ & $\chi_{\mathrm{F}}\left(\mathrm{cm}^{-1} / \mathrm{K}\right)$ & $\chi_{\mathrm{C}}\left(\mathrm{cm}^{-1} / \mathrm{K}\right)$ & $\alpha_{\mathrm{F}}\left(\mathrm{K}^{-1}\right)$ \\
\hline SWCNT & $-18.9 \pm 1.2$ & $-0.029 \pm 0.002$ & $-0.129 \pm 0.01$ & $(1.9 \pm 0.6) \times 10^{-5}$ \\
DWCNT $^{\text {a }}$ & $-14.4 \pm 0.7$ & $-0.021 \pm 0.002$ & $-0.093 \pm 0.01$ & $(2.1 \pm 0.7) \times 10^{-5}$ \\
PRD-166 fibers & $+12 \pm 0.3$ & $-0.154 \pm 0.001$ & $-0.079 \pm 0.001$ & $(9.0 \pm 1.9) \times 10^{-6}$ \\
\hline \hline
\end{tabular}

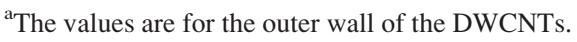

Raman frequencies for the components of the $\mathrm{G}^{\prime}$-band as a function of temperature for both types of nanotube powders. The $\mathrm{G}^{\prime}$-band was seen to shift to lower wavenumber with increasing temperature, which is due to the softening of both the inter-tube interaction and the carbon-carbon bonds, and the temperature-induced perturbation in the electronic structure. ${ }^{21-25}$ It was noted the D- and G-bands also shifted to lower-frequencies with the increase of temperature. However, the $\mathrm{G}^{\prime}$-band was most sensitive to external perturbation and was thus used to measure the coefficient of thermal expansion. The temperature coefficient of the Raman bands, defined as $\mathrm{d} \omega / \mathrm{d} T$, can be obtained from the slope of linear fitting over the entire temperature range. This was found to be $-0.021 \mathrm{~cm}^{-1} / \mathrm{K}$ and $-0.023 \mathrm{~cm}^{-1} / \mathrm{K}$ for the $\mathrm{G}_{1}^{\prime}$ and $\mathrm{G}_{2}^{\prime}$ of the DWCNTs, respectively, while a relatively higher value, of $-0.029 \mathrm{~cm}^{-1} / \mathrm{K}$, was observed for the $\mathrm{G}^{\prime}$-band of the SWCNTs. The higher temperature coefficients of SWCNTs than those of DWCNTs, suggests that the DWCNTs, which were prepared by annealing the $\mathrm{C}_{60} @$ SWCNT peapods at $1573 \mathrm{~K}$, are more stable than the host SWCNTs. ${ }^{26,27}$

The epoxy/CNT composites were also heated up using a hot stage and Fig. 3(b) shows the $\mathrm{G}^{\prime}$-band frequency as a function of temperature for the nanocomposites. The $\omega_{\mathrm{G}^{\prime}}$ of nanotubes in composites is found to decrease approximately linearly with the temperature up to $\sim 373 \mathrm{~K}$, after which an abrupt increase of $\sim 2.5 \mathrm{~cm}^{-1}$ is observed. The temperature coefficient of nanotubes in the composites, defined as $\chi_{\mathrm{C}}=\mathrm{d} \omega_{\mathrm{G}^{\prime}} / \mathrm{d} T$, is obtained by fitting the data linearly in the $293-373 \mathrm{~K}$ range. As the temperature exceeds $403 \mathrm{~K}$, the $\omega_{\mathrm{G}^{\prime}}$ tends to decrease again, but at a lower shift rate.

The glass transition temperature of the epoxy matrix was measured using differential scanning calorimetry (DSC) and the $T_{\mathrm{g}}$ values determined from the first heating are $385 \mathrm{~K}$ and $387 \mathrm{~K}$ for the epoxy/DWCNT and epoxy/SWCNT, respectively (see Fig. S2 in the supplemental material ${ }^{19}$ ). The inflexion in $\omega_{\mathrm{G}^{\prime}}$ in the $383-403 \mathrm{~K}$ range is thus attributed to the glass transition of matrix where the epoxy resin enters into a rubbery state. The close correlation between the Raman band shift data in Fig. 3(b) and DSC data in Fig. S2 suggest that the Raman method could be a useful technique to determine the $T_{\mathrm{g}}$ of an epoxy resin matrix.

When a nanotube composite is heated up, as well as the effect of heating which softens the carbon-carbon bonds and leads to a decrease of $\omega_{\mathrm{G}^{\prime}}$, the mismatch of CTE between the matrix and nanotubes also induces strain which in turn gives rise to a downshift of the $\mathrm{G}^{\prime}$-band. The apparent downshift of $\omega_{\mathrm{G}^{\prime}}$ at a temperature $T, \Delta \omega_{\mathrm{G}^{\prime}}$, in the temperature range of 293-373 K (i.e., before the breakdown of the interface) can be considered as a summation of the thermal and strain effects, which can be expressed as

$$
\Delta \omega_{\mathrm{G}^{\prime}}=\Delta \omega_{\mathrm{G}^{\prime}}^{\mathrm{T}}+\Delta \omega_{\mathrm{G}^{\prime}}^{\mathrm{S}}
$$

where $\Delta \omega_{\mathrm{G}^{\prime}}^{\mathrm{T}}=\chi_{\mathrm{F}} \times \Delta T$ is the shift of $\omega_{\mathrm{G}^{\prime}}$ induced by temperature only and $\Delta \omega_{\mathrm{G}^{\prime}}^{\mathrm{S}}$ is the shift of $\omega_{\mathrm{G}^{\prime}}$ induced by thermal-strain. It is noted that the temperature coefficient of the $G_{2}^{\prime}$ position of the epoxy/DWCNT is significantly lower than that of $G_{1}^{\prime}$. This suggests that the thermal strain induced by the mismatch of CTEs is transferred from the outer wall to the inner wall at a low efficiency, an effect similar to that of the external strain. ${ }^{12}$ To a first approximation, the CTE of epoxy resin, $\alpha_{\mathrm{E}}$, and the CTE of the fillers, $\alpha_{\mathrm{F}}$, can both be assumed to be constants in the temperature range $293-373 \mathrm{~K}$. The parameter $\Delta \omega_{\mathrm{G}^{\prime}}^{\mathrm{S}}$ is then given by

$$
\Delta \omega_{\mathrm{G}^{\prime}}^{\mathrm{S}}=S_{0} \times\left(\alpha_{\mathrm{E}}-\alpha_{\mathrm{F}}\right) \times \Delta T .
$$

The parameter $S_{0}$ is used here instead of $S(0)$ as the matrix is thought to expand isotropically and all nanotubes were parallel to the strain direction. Eq. (2) can be rearranged as

$$
\alpha_{F}=\alpha_{\mathrm{E}}-\left(\chi_{\mathrm{C}}-\chi_{\mathrm{F}}\right) / S_{0},
$$

which allows the CTE of nanotubes to be determined.

The temperature coefficients $\chi_{\mathrm{C}}$ and $\chi_{\mathrm{F}}$, and the strain coefficients $S_{0}$ determined for different nanotubes are listed in Table I. To measure the $\alpha_{\mathrm{E}}$, a PRD-166 alumina fiber which
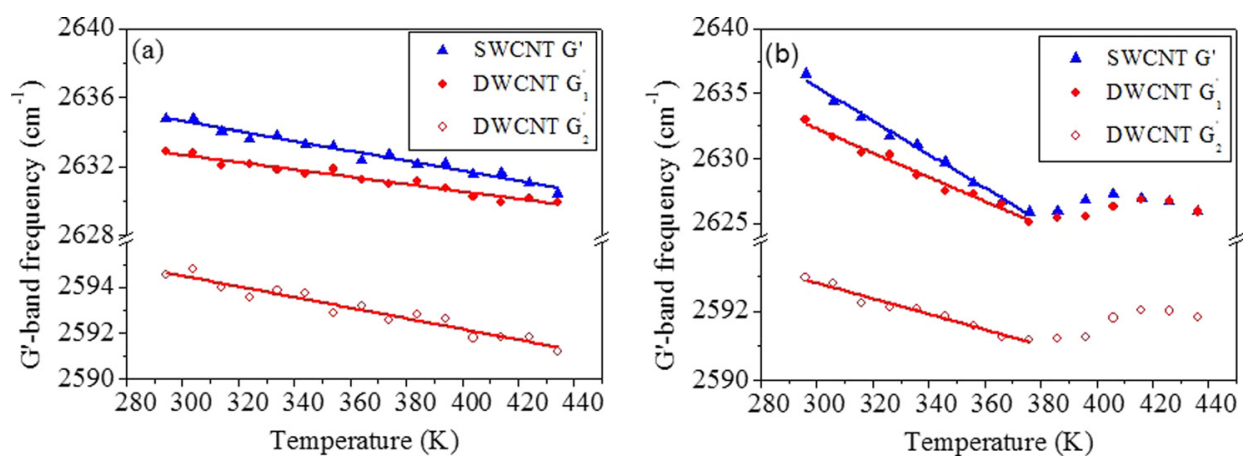

FIG. 3. G'-band frequencies as a function of temperature for: (a) nanotube powders and (b) epoxy/CNT composites. 
has a CTE of $9 \times 10^{-6} \mathrm{~K}^{-1}$ (Ref. 14) was embedded in the same epoxy resin. The alumina fiber exhibits intense and narrow fluorescence bands at around $14400 \mathrm{~cm}^{-1}$, called the R1 and $\mathrm{R} 2$ peaks, due to the presence of a small amount of $\mathrm{Cr}^{3+}$ ion impurity. It is known that the $\mathrm{R}$ bands shift to higher wavenumber when subjected to tensile strain and shift to lower wavenumber upon heating. ${ }^{28,29}$ A series of in-situ fluorescence characterisations of the single alumina fiber and the epoxy/alumina were performed and the variation of the R1 peak position against the strain and temperature was fitted by linear functions (see Figs. S3-S6 in the supplemental material $^{19}$ ). The strain and temperature coefficients obtained for the PRD-166 fiber are also listed in Table I. Substituting the coefficients and CTE of the alumina fiber into Eq. (4) gives a $\alpha_{\mathrm{E}}$ of $7.2 \times 10^{-5} \mathrm{~K}^{-1}$, which agrees well with the data provided by the epoxy resin supplier. This suggests our method is reliable in determining the CTE of thin polymers films, which is particularly useful when bulk samples needed for CTE measurement with other techniques cannot be prepared (e.g., thin insulating layers used in high density electronic packaging). The good agreement also validates our method for determination of the CTE of nanotubes.

Substituting all values into Eq. (4) gives a mean CTE of $2.1 \times 10^{-5} \mathrm{~K}^{-1}$ for the outer wall of the DWCNTs and $1.9 \times 10^{-5} \mathrm{~K}^{-1}$ for the SWCNTs. A large amount of theoretical and experimental work has been carried out to determine the CTE of nanotubes ${ }^{1-6,30}$ but there is no unanimous agreement on even the sign (i.e., expansion or contraction) or the magnitude of the change of nanotube length against the temperature. The positive CTEs found for both DWCNTs and SWCNTs in this study are in qualitative agreement with theoretical work. ${ }^{4,31}$ The thermal expansion behaviour of the DWCNTs found here is also in approximate agreement with the finding by Abe et al., ${ }^{5}$ where a value of $(+0.75 \pm 0.25) \times 10^{-5} \mathrm{~K}^{-1}$ was determined by XRD measurements. It is found that the CTE of DWCNTs was virtually unchanged (within experimental error) compared to the host SWCNTs, a phenomenon which has also been reported before. ${ }^{5}$ It should be stressed; however, research work on thermal expansion has so far been carried out only with nanotube powders. If nanotubes are to be incorporated into polymer composites for practical applications, the processing and environment surrounding the nanotubes may perturb the thermal behaviour of the nanotubes significantly. ${ }^{32}$ Jiang et al. predicted that the CTE of nanotubes can be as high as $2.0 \times 10^{-5} \mathrm{~K}^{-1}$ when strong interactions with substrates are present, ${ }^{2}$ which is close to our experimental values. Our work demonstrates a method of in-situ characterisation of the thermal expansion of nanotubes in composites.

In summary, the CTEs of SWCNTs and DWCNTs have been determined using Raman spectroscopy, by taking into account of both the strain and temperature effects on the Raman band frequency of nanotubes in composites. Both types of nanotubes show a positive thermal expansion, with coefficients of $1.9 \times 10^{-5} \mathrm{~K}^{-1}$ for SWCNTs and $2.1 \times 10^{-5} \mathrm{~K}^{-1}$ for DWCNTs at room temperature. The CTE of the SWCNTs was virtually unchanged upon the introduction of inner wall nanotubes. The method outlined in this study can be used to determine the CTE and $T_{\mathrm{g}}$ of polymers, and may also be extended to evaluate the thermal behaviour of other carbon nanomaterials such as graphene and graphene oxide.

This work was partially supported by Guangdong and Shenzhen Innovative Research Team Program (No. 2011D052, KYPT20121228160843692), National Natural Science Foundation of China (Grant No. 21201175), and R\&D Funds for basic Research Program of Shenzhen (Grant No. JCYJ20120615140007998).

${ }^{1}$ H. Jiang, B. Liu, Y. Huang, and K. C. Hwang, J. Eng. Mater. Technol. 126, 265 (2004)

${ }^{2}$ J.-W. Jiang, J.-S. Wang, and B. Li, Phys. Rev. B 80, 205429 (2009).

${ }^{3}$ Y. K. Kwon, S. Berber, and D. Tománek, Phys. Rev. Lett. 92, 015901 (2004). ${ }^{4}$ P. K. Schelling and P. Keblinski, Phys. Rev. B 68, 035425 (2003).

${ }^{5}$ M. Abe, H. Kataura, H. Kira, T. Kodama, S. Suzuki, Y. Achiba, K. Kato, M. Takata, A. Fujiwara, K. Matsuda, and Y. Maniwa, Phys. Rev. B 68, 041405 (2003).

${ }^{6}$ Y. Maniwa, R. Fujiwara, H. Kira, H. Tou, H. Kataura, S. Suzuki, Y. Achiba, E. Nishibori, M. Takata, M. Sakata, A. Fujiwara, and H. Suematsu, Phys. Rev. B 64, 241402 (2001).

${ }^{7}$ M. S. Dresselhaus, A. Jorio, M. Hofmann, G. Dresselhaus, and R. Saito, Nano Lett. 10, 751 (2010).

${ }^{8}$ S. S. Chen, A. L. Moore, W. W. Cai, J. W. Suk, J. H. An, C. Mishra, C. Amos, C. W. Magnuson, J. Y. Kang, L. Shi, and R. S. Ruoff, ACS Nano 5, 321 (2011).

${ }^{9}$ C. A. Cooper, R. J. Young, and M. Halsall, Composites, Part A 32, 401 (2001).

${ }^{10}$ Z. Li, R. J. Young, and I. A. Kinloch, ACS Appl. Mater. Interfaces 5, 456 (2013).

${ }^{11}$ D. Yoon, Y. W. Son, and H. Cheong, Nano Lett. 11, 3227 (2011).

${ }^{12}$ L. Deng, R. J. Young, S. v. d. Zwaag, and S. Picken, Polymer 51, 2033 (2010).

${ }^{13}$ S. Cui, I. A. Kinloch, R. J. Young, L. Noé, and M. Monthioux, Adv. Mater. 21, 3591 (2009).

${ }^{14}$ X. Yang and R. J. Young, Composites 25, 488 (1994).

${ }^{15}$ C. C. Kao and R. J. Young, Compos. Sci. Technol. 64, 2291 (2004).

${ }^{16}$ M. S. Dresselhaus, G. Dresselhaus, A. Jorio, A. Souza, and R. Saito, Carbon 40, 2043 (2002).

${ }^{17}$ F. Ding, Z. Xu, B. I. Yakobson, R. J. Young, I. A. Kinloch, S. Cui, L. Deng, P. Puech, and M. Monthioux, Phys. Rev. B 82, 041403 (2010).

${ }^{18}$ Z. Xu, H. Li, K. Fujisawa, Y. A. Kim, M. Endo, and F. Ding, Nanoscale 4, 130 (2012).

${ }^{19}$ See supplemental material at http://dx.doi.org/10.1063/1.4864056 for orientation determination, DSC curves of the nanocomposites, and in-situ Raman characterizations of epoxy/alumina fiber composites.

${ }^{20}$ L. Deng, S. J. Eichhorn, C. C. Kao, and R. J. Young, ACS Appl. Mater. Interfaces 3, 433 (2011).

${ }^{21}$ A. Bassil, P. Puech, L. Tubery, W. Bacsa, and E. Flahaut, Appl. Phys. Lett. 88, 173113 (2006).

${ }^{22}$ S. B. Cronin, Y. Yin, A. Walsh, R. B. Capaz, A. Stolyarov, P. Tangney, M. L. Cohen, S. G. Louie, A. K. Swan, M. S. Ünlü, B. B. Goldberg, and M. Tinkham, Phys. Rev. Lett. 96, 127403 (2006).

${ }^{23}$ H. D. Li, K. T. Yue, Z. L. Lian, Y. Zhan, L. X. Zhou, S. L. Zhang, Z. J. Shi, Z. N. Gu, B. B. Liu, R. S. Yang, H. B. Yang, G. T. Zou, Y. Zhang, and S. Iijima, Appl. Phys. Lett. 76, 2053 (2000).

${ }^{24}$ N. R. Raravikar, P. Keblinski, A. M. Rao, M. S. Dresselhaus, L. S. Schadler, and P. M. Ajayan, Phys. Rev. B 66, 235424 (2002).

${ }^{25}$ Y. Zhang, L. Xie, J. Zhang, Z. Wu, and Z. Liu, J. Phys. Chem. C 111, 14031 (2007).

${ }^{26}$ M. He, E. Rikkinen, Z. Zhu, Y. Tian, A. S. Anisimov, H. Jiang, A. G. Nasibulin, E. I. Kauppinen, M. Niemelaü, and A. O. I. Krause, J. Phys. Chem. C 114, 13540 (2010).

${ }^{27}$ L. Song, W. Ma, Y. Ren, W. Zhou, S. Xie, P. Tan, and L. Sun, Appl. Phys. Lett. 92, 121905 (2008).

${ }^{28}$ D. D. Ragan, R. Gustavsen, and D. Schiferl, J. Appl. Phys. 72, 5539 (1992).

${ }^{29}$ R. J. Young, J. Microsc (Oxford, U.K.) 185, 199 (1997).

${ }^{30}$ Y. Yosida, J. Appl. Phys. 87, 3338 (2000).

${ }^{31}$ C. Li and T. W. Chou, Phys. Rev. B 71, 235414 (2005).

${ }^{32} \mathrm{X}$. Yan, T. Itoh, Y. Kitahama, T. Suzuki, H. Sato, T. Miyake, and Y. Ozaki, J. Phys. Chem. C 116, 17897 (2012). 